\title{
Characterization of the Functional MRI Response Temporal Linearity via Optical Control of Neocortical Pyramidal Neurons
}

\author{
Itamar Kahn, ${ }^{1,2 *}$ Mitul Desai, ${ }^{3 *}$ Ulf Knoblich, ${ }^{3 *}$ Jacob Bernstein, ${ }^{3,4}$ Michael Henninger, ${ }^{3,4}$ Ann M. Graybiel, ${ }^{3}$ \\ Edward S. Boyden, ${ }^{3,4}$ Randy L. Buckner, ${ }^{1,2,5}$ and Christopher I. Moore ${ }^{3}$ \\ ${ }^{1}$ Center for Brain Science and Howard Hughes Medical Institute, Harvard University, Cambridge, Massachusetts 02138, ${ }^{2}$ Athinoula A. Martinos \\ Center for Biomedical Imaging, Massachusetts General Hospital, Charlestown, Massachusetts 02129, ${ }^{3}$ McGovern Institute for Brain Research and \\ Department of Brain and Cognitive Sciences and ${ }^{4}$ The MIT Media Laboratory, Synthetic Neurobiology Group and Department of Biological \\ Engineering, Massachusetts Institute of Technology, Cambridge, Massachusetts 02139, and ${ }^{5}$ Departments of Psychiatry and Radiology, \\ Massachusetts General Hospital, Boston, Massachusetts 02114
}

The blood oxygenation level-dependent (BOLD) signal serves as the basis for human functional MRI (fMRI). Knowledge of the properties of the BOLD signal, such as how linear its response is to sensory stimuli, is essential for the design and interpretation of fMRI experiments. Here, we combined the cell-type and site-specific causal control provided by optogenetics and fMRI (opto-fMRI) in mice to test the linearity of BOLD signals driven by locally induced excitatory activity. We employed high-resolution mouse fMRI at 9.4 tesla to measure the BOLD response, and extracellular electrophysiological recordings to measure the effects of stimulation on single unit, multiunit, and local field potential activity. Optically driven stimulation of layer V neocortical pyramidal neurons resulted in a positive local BOLD response at the stimulated site. Consistent with a linear transform model, this locally driven BOLD response summated in response to closely spaced trains of stimulation. These properties were equivalent to responses generated through the multisynaptic method of driving neocortical activity by tactile sensory stimulation, and paralleled changes in electrophysiological measures. These results illustrate the potential of the opto-fMRI method and reinforce the critical assumption of human functional neuroimaging that - to first approximation - the BOLD response tracks local neural activity levels.

\section{Introduction}

The blood oxygenation level-dependent (BOLD) functional MRI (fMRI) signal (Ogawa et al., 1990; Kwong et al., 1992) is widely used to indirectly measure neuronal activity in the human brain. Because the BOLD response measures hemodynamic fluctuations and not underlying electrophysiological signals directly, the relation between neural activity and the BOLD fMRI signal has been studied extensively (Boynton et al., 1996; Dale and Buckner, 1997; Heeger et al., 2000; Miezin et al., 2000; Rees et al., 2000; Logothetis et al., 2001; Mukamel et al., 2005; Viswanathan and Freeman, 2007). With some caveats, most evidence links spike

Received Jan. 2, 2011; revised Aug. 17, 2011; accepted Aug. 29, 2011

Author contributions: I.K., M.D., U.K., A.M.G., E.S.B., R.L.B., and C.I.M. designed research; I.K., M.D., U.K., and J.B. performed research; M.H. contributed unpublished reagents/analytic tools; I.K., M.D., and U.K. analyzed data; I.K., M.D., U.K., A.M.G., E.S.B., R.L.B., and C.I.M. wrote the paper.

This work was supported by the National Science Foundation, National Institutes of Health, the Howard Hughes Medical Institute, and the McGovern Institute for Brain Research. We thank Jessica Cardin, Robert Haslinger, Dominique Pritchett, Jason Ritt, and Jackie Schiller for helpful discussions, Jessica Cardin for technical assistance with histology, and Harvard Center for Brain Science Optical Imaging Core and Steve Turney for assistance with fluorescence imaging.

*I.K., M.D., and U.K. contributed equally to this report.

Correspondence should be addressed to Edward S. Boyden, Randy L. Buckner, or Christopher I. Moore at the above addresses and to Itamar Kahn at his present address: Department of Physiology and Biophysics, Faculty of Medicine, Technion, Israel Institute of Technology, Haifa 31096, Israel. E-mail: esb@media.mit.edu, randy_buckner@harvard.edu, kahn@technion.ac.il, or cim@mit.edu.

D0I:10.1523/JNEUROSCI.0007-11.2011

Copyright $(2011$ the authors $\quad 0270-6474 / 11 / 3115086-06 \$ 15.00 / 0$ rate and the local field potential (LFP) with the BOLD response in the cortex (for reviews, see Heeger and Ress, 2002; Logothetis, 2008). Several studies have further demonstrated that the BOLD response has a roughly linear relation to underlying spiking activity elicited by sensory stimulation (Boynton et al., 1996; Dale and Buckner, 1997; Miezin et al., 2000; Rees et al., 2000; Logothetis et al., 2001).

We built on these prior studies by characterizing the somatosensory neocortical BOLD response in a mouse model that allows precise temporal control of neural activity, an "opto-fMRI" approach. As in other recent demonstrations (Desai et al., 2011; Lee et al., 2010), we combined activation of the microbial opsin channelrhodopsin-2 (ChR2), a light-sensitive nonselective cation channel (Nagel et al., 2003; Boyden et al., 2005), with high-field functional imaging (9.4 tesla fMRI). We employed the Thy1ChR2-yellow fluorescent protein (YFP) mouse, in which ChR2 is expressed in layer $\mathrm{V}$ pyramidal neurons throughout the neocortex (Arenkiel et al., 2007). By targeting primary somatosensory (SI) cortex, we characterized the properties of the BOLD response as a function of pyramidal neuron spiking and tested the hypothesis that the local excitation of pyramidal neurons by optogenetic means recruits a BOLD response that can be modeled with a linear transform from underlying neural activity.

We tested whether a BOLD response evoked by closely temporally spaced short trains of optically driven pyramidal cell activity would summate linearly. We found that the BOLD 
response closely matched local pyramidal neuron activity and added in an approximately linear fashion. These findings both inform our interpretation of the classic sensory driven BOLD neocortical response and provide a quantitative investigation of the opto-fMRI BOLD signal.

\section{Materials and Methods}

Animals. All procedures were conducted in accordance with the National Institutes of Health guidelines and with the approval of the Committee on Animal Care at Massachusetts Institute of Technology. ChR2 transgenic mice (Arenkiel et al., 2007) were purchased from The Jackson Laboratories (line 18, stock no. 007612, strain B6.Cg-Tg(Thy1-COP4/EYFP)18Gfng/J) and subsequently crossed with C57BL/6J mice. Animals used were of either sex. Electrophysiological recordings and fMRI were obtained in separate animals.

Animal anesthesia and surgery. Animals were anesthetized with isoflurane mixed with oxygen for all procedures. During preparation and surgery anesthesia was kept at a level of 1-3\%. Immediately before MRI scanning and electrophysiological recording, the anesthetic level was reduced to $0.5-1 \%$ expired isoflurane. For the MRI experiments, the craniotomy was restricted to a burr hole in the skull. For the electrophysiological recordings, the dura mater was opened after the craniotomy to allow positioning of the optical fiber and recording electrode.

Light stimulation. Light stimulation was generated by a $473 \mathrm{~nm}$ laser (Shanghai Dream Lasers) controlled by a computer. Optical stimulation at $40 \mathrm{~Hz}$ frequency and $8 \mathrm{~ms}$ pulse duration was delivered via a $200 \mu \mathrm{m}$ diameter unjacketed optical fiber with 0.48 numerical aperture (Thorlabs) at a range of $320-470 \mathrm{~mW} \mathrm{~mm}^{-2}$ irradiance at the tip of the fiber, irradiance that can evoke EMG responses when targeting primary motor cortex (Ayling et al., 2009). The fiber was positioned over SI barrel field (SIbf; from bregma: anteroposterior, $-1.5 \mathrm{~mm}$; mediolateral, $2.5 \mathrm{~mm}$ ) at the cortical surface. We computed the dropout in light irradiance using a Monte Carlo simulation (Chow et al., 2010), with a simulated volume of $4 \mathrm{~mm}^{3}, 20 \mu \mathrm{m}^{3}$ voxels, 5 million photons, scattering coefficient in gray matter at $473 \mathrm{~nm}$ of $10 \mathrm{~mm}^{-1}$ (Yaroslavsky, 2002), absorption coefficient of $0.07 \mathrm{~mm}^{-1}$, anisotropy factor of 0.88 , and a uniform laser emission pattern over a half-opening angle of 5 degrees, yielding blue light penetration to layer $\mathrm{V}$ of mouse cortex with about $90 \%$ irradiance loss, chiefly due to scattering of light (Bernstein et al., 2008).

Sensory stimulation. A piezoelectric $(\mathrm{PZ})$ element $(5.0 \mathrm{~cm} \times 0.78 \mathrm{~cm} \times$ $0.18 \mathrm{~cm}$; Noliac) was used to deflect vibrissae. A glass capillary tube was used for extension ( $1 \mathrm{~mm}$ internal diameter and $5 \mathrm{~cm} \mathrm{long}$ ), and a nylon thread was looped through the glass capillary tube. All the posterior vibrissae of the right facial pad were cinched at $\sim 1 \mathrm{~cm}$ from the vibrissal pad. A $40 \mathrm{~Hz}$ sinusoidal wave was used to drive the $\mathrm{PZ}$ element with a peak-to-peak motion amplitude calibrated to $347 \pm 12 \mu \mathrm{m}$ along the dorsoventral direction.

MRI procedures. Mice were fixed to an animal bed (Bruker BioSpin MRI) using a standard tooth piece and ear bars. Breathing rate (Small Animal Monitoring Model 1025, SA Instruments) and expired isoflurane (V9004 Capnograph Series, SurgiVet, Smiths Medical PM) were continuously monitored. Imaging was performed on a 9.4 tesla BioSpec 94/20 USR MRI (Bruker BioSpin MRI) that operates at a maximum gradient strength of $675 \mathrm{mT} / \mathrm{m}$, maximum slew rate $4673 \mathrm{~T} / \mathrm{m} / \mathrm{s}$, and a rise time of $130 \mu \mathrm{s}$. A single transmit and receive surface coil consisting of a single copper loop and etched circuit board ( $20 \mathrm{~mm}$ diameter) was placed over the head. The fiber was placed on or $\leq 50 \mu \mathrm{m}$ from the dura mater. In a subset of mice, the fiber slightly compressed the underlying neocortex, as was seen in the anatomical MRI. These animals produced data identical to that observed in other preparations, suggesting that it did not appreciably impact local cortical responses.

BOLD-contrast signals were acquired using a spin-echo echo-planar sequence (repetition time [TR], $2.5 \mathrm{~s}$; echo time, $12.17 \mathrm{~ms}$; 10 coronal slices, $200 \mu \mathrm{m} \times 200 \mu \mathrm{m} \times 500 \mu \mathrm{m})$. High-resolution $\mathrm{T}_{1^{-}}$and $\mathrm{T}_{2^{-}}$ weighted anatomical images were recorded using a rapid acquisition process with a relaxation enhancement (RARE) sequence in coronal orientations ( 10 coronal slices, $78 \mu \mathrm{m} \times 78 \mu \mathrm{m} \times 500 \mu \mathrm{m})$. Image reconstruction was carried out using ParaVision 5.0 (Bruker BioSpin MRI).
Preprocessing included compensation of slice-dependent time shifts, rigid body correction for motion within and across runs, and smoothing with a full-width half-maximum kernel of $600 \mu \mathrm{m}$. For each mouse in the fMRI component of the study, we conducted interleaved "localizer" and "target" runs. The SIbf region of interest (ROI) identified in the localizer runs was subsequently used to test temporal linearity in the target runs. For each localizer run, the images were detrended and percentage signal change was computed by subtracting, from each voxel's BOLD time series, the temporal average of all off periods for all scans in a session, dividing by the average of the signal across no-stimulation periods and then averaged across all localizer runs. Significant BOLD signal increases were determined by a voxelwise time series correlation of the percentage signal change with the protocol boxcar, delayed by one TR. A family wise error corrected $p$ value of 0.05 was used. To achieve this threshold, only clusters with at least six contiguous voxels and uncorrected $p$ values below 0.005 were considered. The cluster size and $p$ value threshold were determined via Monte Carlo simulations performed in AFNI using AlphaSim (Forman et al., 1995; Xiong et al., 1995).

To estimate the location of activated voxels in SIbf, the EPI volumes were aligned to the T1 structural volumes using the AFNI align_epi_anat. py program. ROIs were identified by overlaying the structural images onto corresponding atlas plates (Paxinos and Franklin, 2001), aligning the anteroposterior coordinates, and scaling the atlas plates along the $x$ and $y$-axes until the borders corresponded.

Electrophysiology. A small craniotomy was made over SIbf. Stimulus control and single unit activity (SUA) data acquisition were performed using software custom written in LabView (National Instruments) and Matlab (The Mathworks). Glass pipette-pulled electrodes (10-14 M $\Omega$ resistance) were filled with saline and lowered under positive pressure while injecting small amplitude current pulses. Cell-attached recording configuration was achieved when a medium resistance seal $(\geq 50 \mathrm{M} \Omega)$ was formed and spikes were observed (MultiClamp 700B, Molecular Devices). LFP and multiunit activity (MUA) recordings were obtained using 16 contacts ( $15 \mu \mathrm{m}$ diameter spaced $100 \mu \mathrm{m}$ apart) laminar electrodes (Neuronexus Technologies) connected to a Cheetah32 data acquisition system (Neuralynx). Recordings were performed at $0.1-9000 \mathrm{~Hz}$ filter settings and separated into LFP and MUA signals post hoc.

Electrophysiology analysis. Cell-attached recording data were bandpass filtered between 600 and $6000 \mathrm{~Hz}$, and spike times were extracted where the potential exceeded 10 standard deviations of the signal calculated across the entire block. The MUA data were extracted from laminar recordings by bandpass filtering the signal between 600 and $6000 \mathrm{~Hz}$, and spike times were extracted where the potential exceeded 4 standard deviations of the signal calculated across the entire block. All firing rate data were binned at $100 \mathrm{~ms}$. The LFP amplitudes were calculated by subtracting any slow drift in the potential (piecewise linear fit to the potential at the beginning of each trial) and averaging the negative of the signal during the entire stimulus presentation. For LFP power, the signal was lowpass filtered at $300 \mathrm{~Hz}$. Spectrograms were computed for each trial using a $250 \mathrm{~ms}$ Hamming window and frequencies from 7 to $200 \mathrm{~Hz}$. Values reported represent the average over all frequencies and the entire stimulus presentation time.

Statistical procedures. We tested the data for normality with the Lilliefors test (Lilliefors, 1967). The electrophysiological measures exhibited normal distributions, but the fMRI BOLD data exhibited non-normal distributions $(p<0.005)$. Therefore, we conducted nonparametric tests throughout (Wilcoxon signed rank and rank-sum two-tail tests). Goodness-of-fit $\left(R^{2}\right)$ measures were derived using least-squares regression of the averaged time series from each animal against the predicted response.

Histology. Mice ( $n=4$; two from the MRI experiment and two from the electrophysiology experiment) were transcardially perfused with 100 mM phosphate buffer (PB) followed by $4 \%$ formaldehyde in $\mathrm{PB}$, and brains were postfixed for $18 \mathrm{~h}$ at $4^{\circ} \mathrm{C}$. Free-floating sections $(50 \mu \mathrm{m})$ were cut using a freezing microtome (American Optical Model 820) mounted on glass slides with Vectashield (Vector Laboratories) and coverslipped. Spread and labeling efficiency were estimated by examination of $50 \mu \mathrm{m}$ coronal sections near the SIbf for the presence of YFP using a Zeiss LSM 

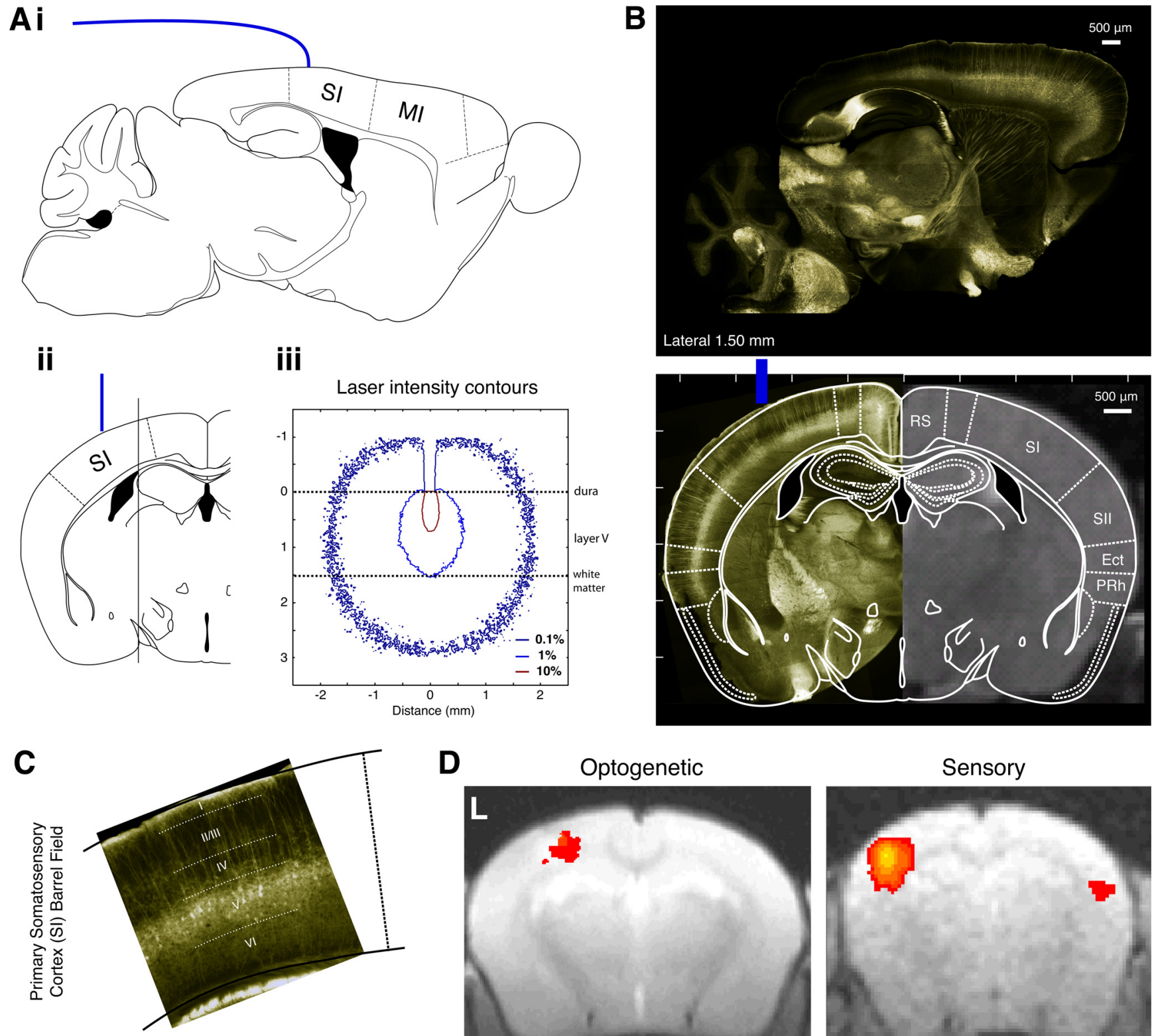

D
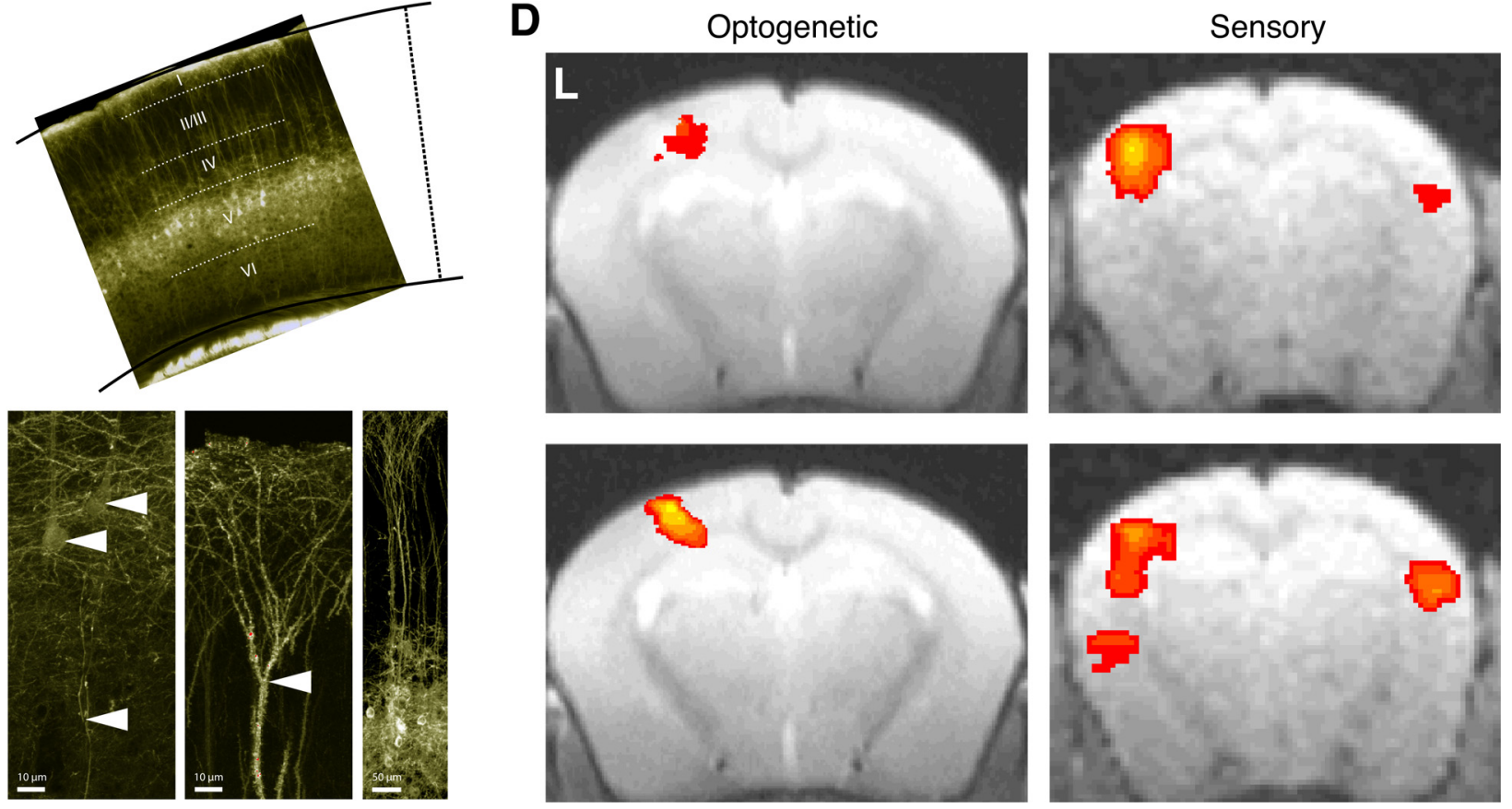

Figure 1. Optogenetic fMRI. Neural activation of layer V ChR2-expressing neurons by light stimulation evokes a reliable fMRI response that is equivalent to somatosensory response evoked by vibrissa deflection. A, Opto-fMRI experimental design. Atlas (Paxinos and Franklin, 2001) sagittal (i) and coronal (ii) sections depict the placement of the fiber optic (blue line) over primary somatosensory cortex (SI) barrel field. Monte Carlo simulation of laser intensity contours are plotted (iii), demonstrating that light intensity at $750 \mu$ m was $10 \%$, power sufficient to induce action potentials in layer V pyramidal neurons in vitro. MI, Primary motor cortex. B, Wide-field fluorescence depicts ChR2 expression of primarily layer V pyramidal neurons in a transgenic Thy 1-ChR2-YFP mouse. Sagittal slice depicts expression throughout the neocortex, and an atlas section is superimposed on representative histological and anatomical MRI coronal sections, depicting the site of stimulation for a representative animal. C, A characteristic expression is shown demonstrating fluorescence in layer V pyramidal neurons (top). Maximum intensity projection of confocal laser scanning microscopy demonstrates ChR2 expression (bottom). Denoted by white triangles are apical dendrites approaching the surface, the cell bodies, and axons. $\boldsymbol{D}$, "Localizer" runs consisted of 16 repetitions of $15 \mathrm{~s} \mathrm{light-pulses} \mathrm{or} \mathrm{deflections} \mathrm{of} \mathrm{the} \mathrm{mystacial} \mathrm{vibrissae} \mathrm{followed} \mathrm{by} 15 \mathrm{~s}$ of no stimulation. A statistical parametric map of a positive BOLD response to light stimulation ( $p<0.05$ corrected for multiple comparisons) is overlaid on the average fMRI BOLD image of the sequential cross-sections demonstrating the response for the optical drive in a typical animal ("Optogenetic"). A similar BOLD response was observed to vibrissa deflections ("Sensory"). 
A
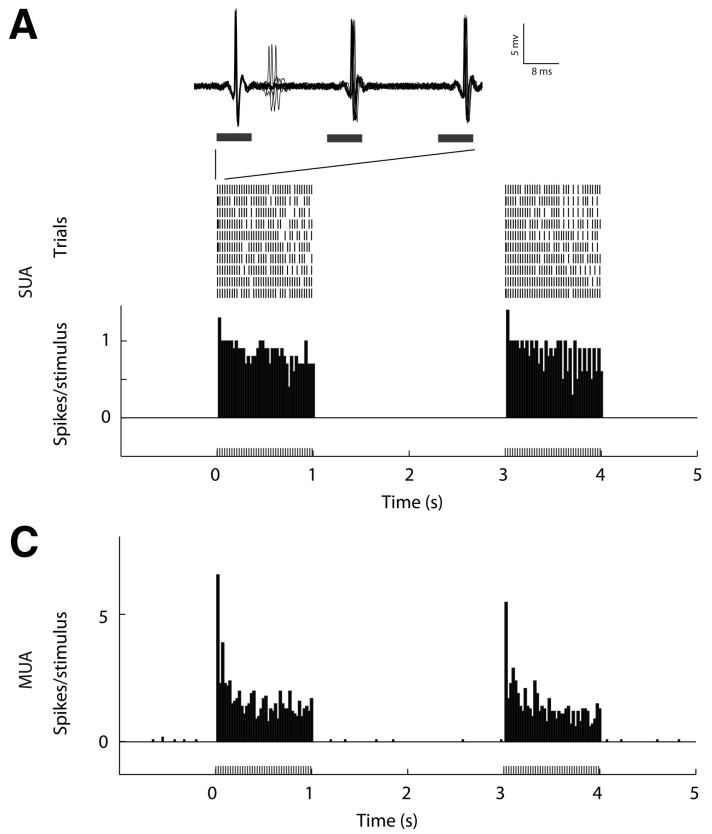

$\mathbf{E}$

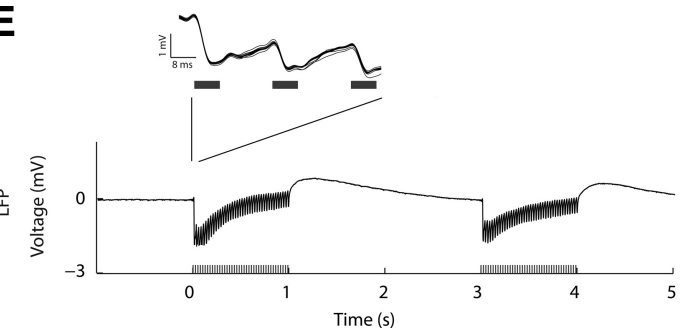

B
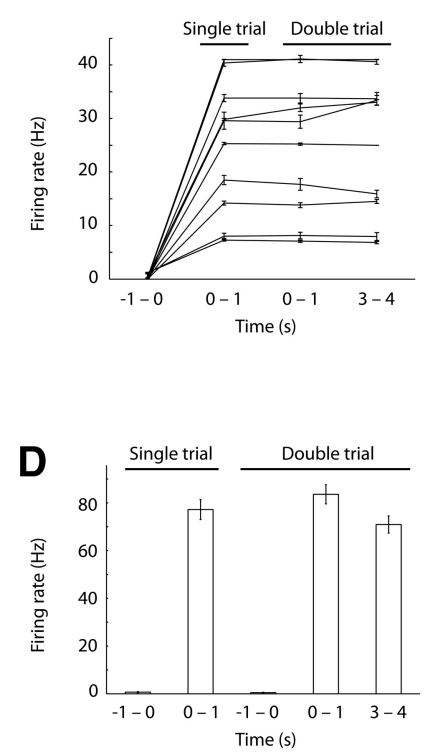

$\mathbf{F}$

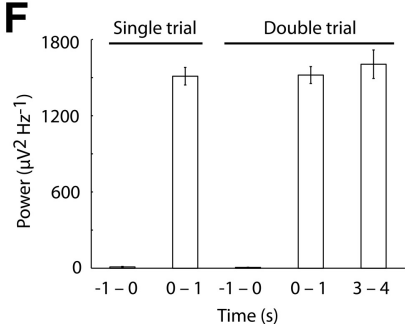

Figure 2. Precisely evoked spike and local field potential activity for $1 \mathrm{~s}$ trains of optical stimulation of primary somatosensory cortex. A, Activity of a representative single neuron recorded juxtacellularly is depicted (single-unit activity, SUA). Raster plot (top) and peri-stimulus time histogram (PSTH; bottom) show equivalent spike responses for the two trains of $1 \mathrm{~s} \mathrm{stimulation.} \mathrm{Overlaid}$ voltage traces during the first three light pulses (inset) show highly reliable spike times and shape. $\boldsymbol{B}$, SUA shown for individual neurons, demonstrating that although firing rate was variable between neurons, each neuron showed equivalent firing rate for $1 \mathrm{~s}$ train of light pulses in all conditions. C, PSTH of multiunit activity (MUA) recorded using a laminar electrode is depicted. Responses are averaged from two contacts (depths of 700 and $800 \mu \mathrm{m}$ ). D, Similar to SUA, MUA responses were above baseline and equivalent when comparing the $1 \mathrm{~s}$ optical stimulation intervals. $\boldsymbol{E}$, Local field potential (LFP) modulation was observed for each light stimulation train. A representative voltage trace is shown for one trial. The consistency of the response across individual trials is shown by the high overlap of individual traces (inset). $\boldsymbol{F}$, Similar to SUA and MUA, power modulation was equivalent across the 1 sintervals.

5 Pascal (Carl Zeiss light microscopy) in wide-field fluorescence and laser scanning confocal modes.

\section{Results}

The SIbf was chosen as the optical stimulation site. Figure 1, $A$ and $B$, shows typical fiber optic placement. The light irradiance at the tip of the fiber was at least $300 \mathrm{~mW} \mathrm{~mm}^{-2}$, such that at layer $\mathrm{V}$ about $10 \%$ of the light was present (Fig. 1 Aiii), a power level that fully activates ChR2 (Boyden et al., 2005). To confirm expression of the ChR2-YFP fusion protein under the control of the Thyl promoter in the stimulated site, we carried out histological experiments in four mice. Robust ChR2-YFP expression was observed in layer $\mathrm{V}$ pyramidal neurons (Fig. $1 B, C$ ) with extensive expression in apical dendrites and cell bodies. The pattern was consistent with previously reported expression in Thy1 promoter-driven lines (Feng et al., 2000; Arenkiel et al., 2007; Ayling et al., 2009).

We first tested whether a BOLD response could be observed in response to optical stimulation in neurons expressing ChR2 and the extent to which optical drive spatially corresponded to sen- sory driven responses. In each animal participating in the fMRI experiment, $2-8$ localizer runs were intermixed with target runs. In each localizer run, either light pulses (eight animals) or vibrissa deflections (four animals) were applied for $15 \mathrm{~s}$ followed by $15 \mathrm{~s}$ of no stimulation, and this sequence was repeated 16 times. BOLD responses were observed in SIbf for the optical and sensory conditions (see Fig. $1 D$ for typical optogenetic and sensory responses). Optically driven BOLD responses extended over a volume of $0.65 \pm 0.29 \mathrm{~mm}^{3}$ (mean $\pm \mathrm{SD}$; eight animals), consistent with the emitted light irradiance. Similarly, sensory driven BOLD responses extended over a volume of $0.60 \pm 0.11 \mathrm{~mm}^{3}$ (four animals).

Dale and Buckner (1997) observed that the BOLD response summated in an approximately linear fashion to pairs of brief visual stimuli (1 s) spaced by as little as $2 \mathrm{~s}$. We sought to test the assertion that subtraction of $(n) 1 \mathrm{~s}$ trains from $(n+1)$ $1 \mathrm{~s}$ trains of activity would yield a response equivalent to a single $1 \mathrm{~s}$ train. Employing cell-attached juxtacellular recording from single units (SUA; 5 animals) as well as MUA and LFP recording with 16-channel laminar electrodes (1 animal) we observed an equivalent level of activity by $1 \mathrm{~s}$ periods of stimulation in both a single ( $1 \mathrm{~s}$ interval of stimulation) and double (two $1 \mathrm{~s}$ intervals spaced by $2 \mathrm{~s}$ ) trial conditions (Fig. 2). We next extracted the BOLD time series from voxels identified in localizer runs for all animals (Fig. 3). The single and double trial conditions demonstrated an approximately linear increase, where a subtraction of the single trial from the double trial yielded a time series matching the single trial condition (Fig. $3 A$; six animals). To test the generality of this result, we asked whether this pattern held when multiple $1 \mathrm{~s}$ trains were compared. We compared in a separate experiment (two animals) optical drive of five (quintuple) to six (sextuple) $1 \mathrm{~s}$ trains. The computed subtraction of these conditions yielded a response similar to that observed for the single $1 \mathrm{~s}$ train (Fig. $3 B$ ). To provide a bridge to BOLD responses observed in human $\mathrm{AMRI}$, we compared the optical somatosensory-evoked responses to multisynaptic sensory-evoked responses such as those generated by vibrissal deflections. Both the single - double (two animals; Fig. 3C) and quintuple - sextuple (two animals; Fig. 3D) trial conditions exhibited what appeared to be linear increases.

We quantified the temporal linearity of the BOLD response using a variety of metrics. First, we verified that the integrated BOLD response of the $(n+1)$ trials produced a significantly greater response relative to $(n)$ trials in the optical and sensory driven responses (Wilcoxon signed rank paired two-tailed test; Optogenetic (optical drive): single - double, $p<0.0001$, and quintuple - sextuple, $p<0.05$; Sensory (sensory drive): single double, $p<0.01$, and quintuple - sextuple, $p<0.05$ ). Second, we 

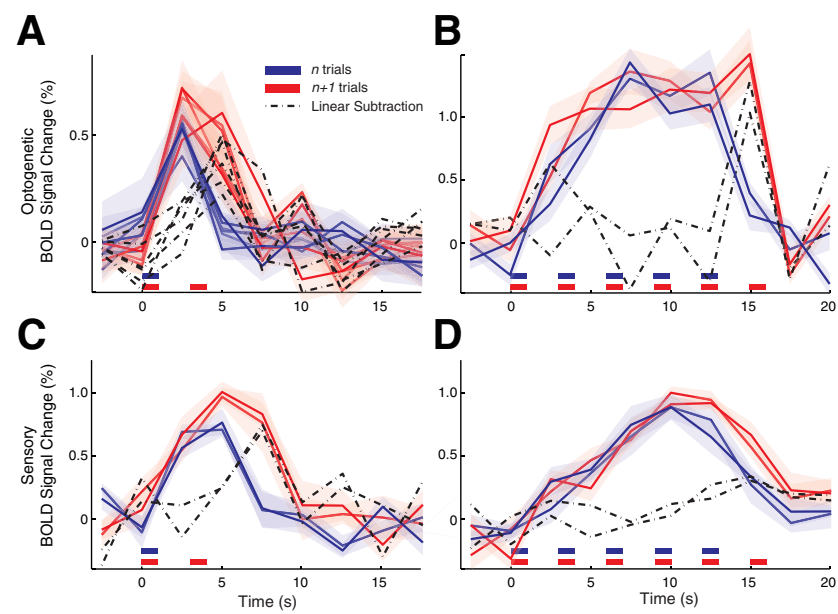

Figure 3. The BOLD response shows linear temporal summation for closely spaced short trains of stimulation. For each animal, time series were extracted from a ROl identified in independent localizer runs. The linear response can be qualitatively observed by subtracting the $n$ from the $n+1$ conditions (dashed line). $\boldsymbol{A}$, Optical stimulation evoked equivalent responses across multiple independent $1 \mathrm{~s}$ trains. Individual time series ( $n=6$ animals) of the BOLD fMRI response to one 1 s interval (single) and two trains of 1 s intervals (double) with an interstimulus interval of $2 \mathrm{~s}$. Data are expressed as mean \pm SEM (across individual runs in each animal). $\boldsymbol{B}$, Five $1 \mathrm{~s}$ (quintuple) and six $1 \mathrm{~s}$ trains (sextuple) show an equivalent temporal linearity response (two animals) to that observed in $\boldsymbol{A}$. C, D, Sensory stimulation yielded equivalent responses in the single - double (two animals) and quintuple - sextuple (two animals) experiments.

confirmed that the temporal evolution of a single trial response yielded a response equivalent to a double trial by testing the goodness of fit of the double trial to the summation of a single trial and a single trial shifted by $3 \mathrm{~s}$ (Fig. $4 A, B$; Optogenetic: $R^{2}=0.64$, $F_{(1,23)}=38.94, p<0.0001$; Sensory: $R^{2}=0.88, F_{(1,7)}=44.60, p<$ $0.001)$. Third, we computed the ratio between the responses of the $(n+1)$ and $(n)$ trial conditions (Fig. 4C,D). For the single - double conditions the BOLD response signal was integrated over the 2.5-12.5 s period for the optical and sensory driven responses; for SUA and MUA the total number of spikes was counted, and for LFPs the total power was calculated. The electrophysiological measures yielded a double to single response ratio close to 2: $1.99 \pm 0.02$ (mean \pm SEM) for SUA, $2.05 \pm 0.07$ for MUA, and $2.05 \pm 0.04$ for LFP, and a similar ratio held for the BOLD responses (Optogenetic: $1.97 \pm 0.25$; Sensory: $1.70 \pm$ $0.18)$. Similarly, for the quintuple - sextuple conditions BOLD integrated responses were computed over the 2.5-22.5 s period for the optical and sensory driven responses, yielding a response ratio close to 1.2 (Optogenetic: $1.25 \pm 0.08$; Sensory: $1.25 \pm$ $0.08)$. Finally, we tested whether the linear model holds by computing the goodness of fit of the subtraction of $(n)$ from $(n+1)$ trial conditions time series to the single trial response. A hemodynamic response function (HRF) was fitted to the single trial condition of the optical and sensory drive separately by a mixture of two Gamma functions $\left(f_{1}\right.$ and $f_{2}$ ) fitting a delay of the response and undershoot, dispersion of response and undershoot, and ratio of response to undershoot [probability density function of Gamma distribution with shape $(h)$ and scale $(l)$ parameters; Optogenetic: $f_{1}(h=5.55, l=0.34), f_{2}(h=10.42, l=0.13)$; Sensory: $\left.f_{1}(h=5.05 ; l=0.16), f_{2}(h=8.33 ; l=13)\right]$. The resultant HRF was tested for goodness of fit to the subtracted responses for the single - double and quintuple - sextuple. In the optical stimulation condition, the subtracted responses were well fitted by the HRF (Fig. $4 E$; single - double: $R^{2}=0.53, F_{(1,29)}=31.06$, $p<0.0001$; quintuple - sextuple: $R^{2}=0.68, F_{(1,9)}=16.86, p<$
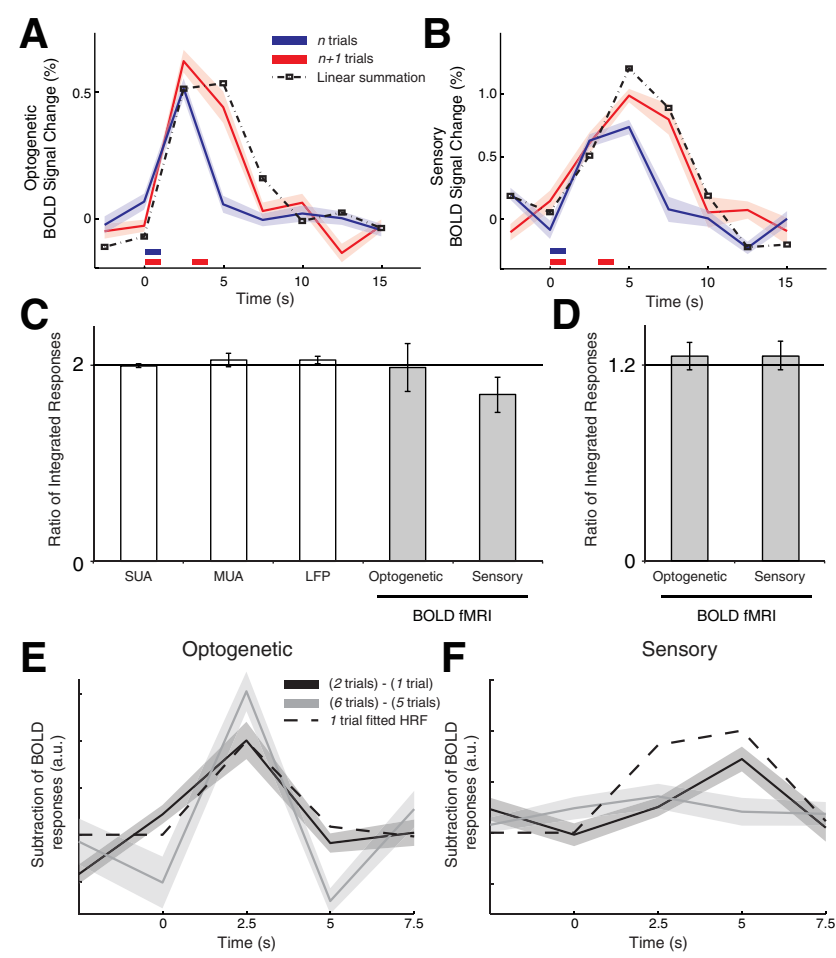

Figure 4. BOLD temporal linearity. $\boldsymbol{A}$, Optical stimulation evoked equivalent responses across multiple independent $1 \mathrm{~s}$ trains. Average time series ( $n=6$ animals) of the BOLD fMRI response to one 1 s interval (single) and two trains of 1 s intervals (double) with an interstimulus interval of $2 \mathrm{~s}$. Data are expressed as mean \pm SEM (individual runs across all animals). Linear summation of the single trial to a 3 s shifted single trial (dashed line) provides a qualitative estimate of the temporal summation. $\boldsymbol{B}$, Linear summation similarly holds for sensory stimulation. $C, D$, The temporal linearity response was quantified by dividing the response for the $(n+$ 1) trials condition by the $(n)$ trials condition. $\boldsymbol{E}, \boldsymbol{F}$, A hemodynamic response function fitted to the single trial condition is plotted against the subtracted responses for the single - double and quintuple - sextuple trial conditions. Optogenetic refers to optical drive, Sensory to sensory drive.

0.005). In the sensory stimulation condition, only the single double subtraction resulted in a reliable goodness-of-fit to the HRF (Fig. 4F; single - double: $R^{2}=.585, F_{(1,9)}=11.2778, p=$ 0.01 ; quintuple - sextuple: $R^{2}=0.2773 F_{(1,9)}=3.0040, p=0.12$ ). Thus, consistent with spike activity in individual neurons, short trains of activity, when separated in time, linearly summated in the BOLD response. Furthermore, linearity of the hemodynamic response held for optogenetic drive consistently, while it failed for sensory drive in the quintuple - sextuple condition.

\section{Discussion}

We applied optogenetic stimulation to control the precise timing of neocortical pyramidal neuron activity and tested the spike, LFP, and BOLD signal responses to this stimulation. We demonstrate that when regular spiking pyramidal cells in layer $\mathrm{V}$ of the sensory neocortex were driven by stimulation of light-activated cation channels, a hemodynamic response was evoked reliably. We recorded SUA, MUA, and LFP activity to test whether the BOLD response followed the linear transform model. We observed a linear summation of the BOLD response for temporally spaced short trains of optical stimulation that was proportional to the cumulative neural activity recorded as SUA, MUA, and LFP signals.

A core assumption of brain imaging with fMRI using the BOLD-contrast mechanism is that local changes of neural activity elicit a hemodynamic response and that the hemodynamic response is approximately linearly related to the underlying neural 
activity (Boynton et al., 1996; Dale and Buckner, 1997; Miezin et al., 2000; Rees et al., 2000; Logothetis et al., 2001; Heeger and Ress, 2002). Our results suggest that-to first approximation-this assertion is supported. Optically driven BOLD responses demonstrated temporal linear summation following closely temporally spaced trains of stimulation. Furthermore, our data indicate that similar analysis assumptions can be applied to opto-fMRI responses generated by direct optical activation of pyramidal neurons. Our findings suggest that a rodent model may be a valid approach to explore what inferences can be made about underlying neural activity from the indirect BOLD response.

Our results using opto-fMRI to probe the BOLD response suggest a general approach to study systematically the relation between local changes in neural activity and the BOLD response recorded by fMRI. The opto-fMRI approach we employed is similar to that taken by Desai et al. (2011) and Lee et al. (2010), who used opto-fMRI to study network connectivity, and activated CaMKII $\alpha$-expressing excitatory neurons that were virally transduced. We used the Thy 1 transgenic mouse model expressing ChR2, providing an effective system for studying the relation between neural activity and fMRI. Critically, we observed a deviation from linearity using sensory drive when multiple $1 \mathrm{~s}$ intervals of stimulation were applied. Failure of linearity can, in principle, result from failure of linearity between the stimulus and neural responses or neural responses and the hemodynamic response. The present results eliminate the latter possibility. By focusing on understanding the local response, we have probed the neural and BOLD responses under conditions typically employed in human imaging, the summation of short duration, closely spaced, event-related signals.

\section{References}

Arenkiel BR, Peca J, Davison IG, Feliciano C, Deisseroth K, Augustine GJ, Ehlers MD, Feng G (2007) In vivo light-induced activation of neural circuitry in transgenic mice expressing channelrhodopsin-2. Neuron 54:205-218.

Ayling OG, Harrison TC, Boyd JD, Goroshkov A, Murphy TH (2009) Automated light-based mapping of motor cortex by photoactivation of channelrhodopsin-2 transgenic mice. Nat Methods 6:219-224.

Bernstein JG, Han X, Henninger MA, Ko EY, Qian X, Franzesi GT, McConnell JP, Stern P, Desimone R, Boyden ES (2008) Prosthetic systems for therapeutic optical activation and silencing of genetically-targeted neurons. Proc Soc Photo Opt Instrum Eng 6854:68540H.

Boyden ES, Zhang F, Bamberg E, Nagel G, Deisseroth K (2005) Millisecondtimescale, genetically targeted optical control of neural activity. Nat Neurosci 8:1263-1268.

Boynton GM, Engel SA, Glover GH, Heeger DJ (1996) Linear systems analysis of functional magnetic resonance imaging in human V1. J Neurosci 16:4207-4221.

Chow BY, Han X, Dobry AS, Qian X, Chuong AS, Li M, Henninger MA, Belfort GM, Lin Y, Monahan PE, Boyden ES (2010) High-performance genetically targetable optical neural silencing by light-driven proton pumps. Nature 463:98-102.

Dale AM, Buckner RL (1997) Selective averaging of rapidly presented individual trials using fMRI. Hum Brain Mapp 5:329-340.

Desai M, Kahn I, Knoblich U, Bernstein J, Atallah H, Yang A, Kopell N,
Buckner RL, Graybiel AM, Moore CI, Boyden ES (2011) Mapping brain networks in awake mice using combined optical neural control and fMRI. J Neurophysiol 105:1393-1405.

Feng G, Mellor RH, Bernstein M, Keller-Peck C, Nguyen QT, Wallace M, Nerbonne JM, Lichtman JW, Sanes JR (2000) Imaging neuronal subsets in transgenic mice expressing multiple spectral variants of GFP. Neuron 28:41-51.

Forman SD, Cohen JD, Fitzgerald M, Eddy WF, Mintun MA, Noll DC (1995) Improved assessment of significant activation in functional magnetic resonance imaging (fMRI): use of a cluster-size threshold. Magn Reson Med 33:636-647.

Heeger DJ, Ress D (2002) What does fMRI tell us about neuronal activity? Nat Rev Neurosci 3:142-151.

Heeger DJ, Huk AC, Geisler WS, Albrecht DG (2000) Spikes versus BOLD: what does neuroimaging tell us about neuronal activity? Nat Neurosci 3:631-633.

Kwong KK, Belliveau JW, Chesler DA, Goldberg IE, Weisskoff RM, Poncelet BP, Kennedy DN, Hoppel BE, Cohen MS, Turner R, Cheng HM, Brady TJ, Rosen BR (1992) Dynamic magnetic resonance imaging of human brain activity during primary sensory stimulation. Proc Natl Acad Sci U S A 89:5675-5679.

Lee JH, Durand R, Gradinaru V, Zhang F, Goshen I, Kim DS, Fenno LE, Ramakrishnan C, Deisseroth K (2010) Global and local fMRI signals driven by neurons defined optogenetically by type and wiring. Nature 465:788-792.

Lilliefors HW (1967) On the Kolmogorov-Smirnov test for normality with mean and variance unknown. J Am Stat Assoc 62:399-402.

Logothetis NK (2008) What we can do and what we cannot do with fMRI. Nature 453:869-878.

Logothetis NK, Pauls J, Augath M, Trinath T, Oeltermann A (2001) Neurophysiological investigation of the basis of the fMRI signal. Nature 412:150-157.

Miezin FM, Maccotta L, Ollinger JM, Petersen SE, Buckner RL (2000) Characterizing the hemodynamic response: effects of presentation rate, sampling procedure, and the possibility of ordering brain activity based on relative timing. Neuroimage 11:735-759.

Mukamel R, Gelbard H, Arieli A, Hasson U, Fried I, Malach R (2005) Coupling between neuronal firing, field potentials, and FMRI in human auditory cortex. Science 309:951-954.

Nagel G, Szellas T, Huhn W, Kateriya S, Adeishvili N, Berthold P, Ollig D, Hegemann P, Bamberg E (2003) Channelrhodopsin-2, a directly lightgated cation-selective membrane channel. Proc Natl Acad Sci U S A 100:13940-13945.

Ogawa S, Lee TM, Nayak AS, Glynn P (1990) Oxygenation-sensitive contrast in magnetic resonance image of rodent brain at high magnetic fields. Magn Reson Med 14:68-78.

Paxinos G, Franklin KBJ (2001) The mouse brain in stereotaxic coordinates, Ed 2. San Diego: Academic.

Rees G, Friston K, Koch C (2000) A direct quantitative relationship between the functional properties of human and macaque V5. Nat Neurosci 3:716-723.

Viswanathan A, Freeman RD (2007) Neurometabolic coupling in cerebral cortex reflects synaptic more than spiking activity. Nat Neurosci 10:1308-1312.

Xiong J, Gao J, Lancaster J, Fox P (1995) Clustered pixels analysis for functional MRI activation studies of the human brain. Hum Brain Mapp 3:287-301.

Yaroslavsky AN, Schulze PC, Yaroslavsky IV, Schober R, Ulrich F, Schwarzmaier HJ (2002) Optical properties of selected native and coagulated human brain tissues in vitro in the visible and near infrared spectral range. Phys Med Biol 47:2059-2073. 